\title{
Progress of Nonlinear Dust Acoustic Waves in Dust Plasma of Opposite Polarity
}

\author{
H. H. Salah
}

\author{
Department of Physics, Faculty of Science (Girls Branch), Al-Azher University, Nasr City, Cairo, \\ Egypt
}

ABSTRACT

hhashem81@yahoo.com

The propagation of dust-acoustic solitary waves in an magnetized collisionless plasma consisting of positive and negative dust, electrons and ions with Boltzmann distribution are examined. For nonlinear dust acoustic waves $(D A W s)$, a reductive perturbation method was employed to obtain the Kortewege-de Vries (KdV) equation for the first-order potential. As the wave amplitude enlarged, the width and velocity of the wave deviate from the prediction of the KdV equation. Higher order analysis of the perturbed (KdV) equation was used to the fifth-order dispersion term. The effects of higher-order corrections on dust acoustic solitary structures are studied and discussed.

Keywords: : Dust acoustic waves Kortewege-de Vries (KdV) Equation; Solitary solution.

\section{INTRODUCTION}

Dusty plasma is an ionized gas containing small particles of solid matter which acquire a large electric charge by collecting electrons and ions from the plasma, which has been observed widely in astrophysical plasma and environment, such as plasmas in inplanetary rings, in circum-solar dust grains, in the interplanetary medium, in cometary comae and tails, in asteroid zones, in mesosphere and magnetosphere, and in interstellar molecular clouds $[1,2]$. Because of the involving of the charged dust grains in plasmas, different types of collective processes exist and very rich wave modes can be excited in dusty plasmas such as, DA waves $[3,4]$ dust ion acoustic (DIA) waves [5,6], dust-lattice (DL) waves $[7,8]$. The charging of dust grains occurs due to a variety of processes [9-11]. Mamun and Shukla [12] have considered dusty plasma model, which consists of positive and negative dust only, and have theoretically investigated the properties of linear and nonlinear electrostatic waves in such a dusty plasma. The dusty plasma model of Mamun and Shukla [12] is only valid if a complete depletion of the background electrons and ions is possible, and both positive and negative dust fluids are cold. Recently, El Wakil [13] investigated theoretically the higherorder contributions to nonlinear dust-acoustic waves that propagates in a mesospheric dusty plasma with a completely depletion of background (electrons and ions). However, in most space dusty plasma systems a complete depletion of the background electrons and ions is not possible [14-17] and the positive dust component is of finite temperature [18,19]. Later, Attia et. al. [20] investigated the higher order effects of positive and negative dust charge fluctuation on the propagation of dust ion acoustic waves (DIAWs) in a weakly inhomogeneous, weakly coupled, collisionless and unmagnetized mesospheric dusty Plasma consists of four components dusty plasma.
The evolution of small but finite-amplitude solitary structures in plasma systems, studied by means of $\mathrm{KdV}$ equation, is of considerable interest in plasma dynamics. These equations derived from perturbation methods such as the reductive perturbation theory (RPT) [21]. It was found that the RPT is based on the small wave amplitude, the first order solution would underestimate the amplitude of the soliton by as much as $20 \%$. As the wave amplitude enlarge, the soliton width and velocity deviate from the prediction of $\mathrm{KdV}$ equation. Therefore, To overcome this deviation, higher-order corrections must be taken into account [22-26]. So, our motive here is to study the effect of the higherorder dispersion term on the propagation velocity, the amplitude, and the width in four component plasma. The organization of the paper is as follows: In Section 2 we present the basic set of fluid equations governing our plasma model. Section 3 contains the nonlinear analysis for IAWs. In Section 4, Higher order solution is obtained. Finally, discussions and conclusions are given in Sections 5.

\section{BASIC EQUATIONS}

Let us consider a homogeneous system of a magnetized collisionless plasma consisting of a fourcomponent dusty plasma with massive, micron-sized, positively, negatively dust grains and nonthermal elcetron and ion. This study based on the condition that, the negative dust particles are much more massive than positive ones [27,28]. The dynamics of the nonlinear DA waves in the presence of an external magnetic field $B_{0}=\vec{e}_{x} B_{0}$ of such system is governed by:

$$
\begin{aligned}
& \frac{\partial n}{\partial t}+\nabla \cdot(n \mathbf{u})=0, \\
& \rho\left(\frac{\partial \mathbf{u}}{\partial t}+(\mathbf{u} \cdot \nabla) \mathbf{u}\right)+\nabla \boldsymbol{\varphi}+\frac{\sigma_{d}}{n} \nabla \mathbf{p}-\left(\mathbf{u} \times \mathbf{\Omega}_{1} \vec{e}_{x}\right)=0,
\end{aligned}
$$


$2.1 \frac{\partial \mathbf{p}}{\partial t}+\mathbf{u} \nabla \mathbf{p}+\gamma \mathbf{p} \nabla \mathbf{u}=0$,

for Positive dust plasma and

$\frac{\partial N}{\partial t}+\nabla \cdot(N v)=0$

$\frac{\partial \mathbf{v}}{\partial t}+\mathbf{v} \cdot \nabla \mathbf{v}-\nabla \boldsymbol{\varphi}+\left(\mathbf{v} \times \mathbf{\Omega}_{2} \vec{e}_{x}\right)=0$,

for negative dust plasma.

Equations (2.1) and (2.2) are supplemented by Poisson's equation:

$\nabla^{2} \phi=N-\mu n+\mu_{e} n_{e}-\mu_{i} n_{i}$

$n_{e}=e^{\sigma_{e} \phi}$

$n_{i}=e^{-\phi}$.

In the above equations $n$ and $u$ are the density and velocity of positively charged dusty grains while $N$ and $v$ are the density and velocity of negatively charged dusty grains, $n_{e}$ and $n_{i}$ are the density of electrons and ions, $\phi$ and $p$ are the electric potential of dust fluid and the thermal pressure of the positively charged dust fluid, respectively. Here $n$ and $N$ are normalized by their equilibrium values $n_{o}$ and $N_{o} \cdot u$ and $v$ are normalized by $C_{s}=\sqrt{\rho} V_{T}$,

$\rho=Z_{n} m_{1} / Z_{p} m_{2}, \quad V_{T}=\left(Z_{p} k_{B} T_{i} / m_{1}\right)^{\frac{1}{2}}, \quad Z_{p}\left(Z_{n}\right)$, represents the number of the positive (negative) charges on the dust grain surface, $m_{1}\left(m_{2}\right)$ represents the mass of the positive (negative) dust particle, $k_{B}$ is the Boltzmann constant, $T_{i}$ is the temperature of the ions, $p$ is normalized by $n_{o} k_{B} T_{p}$, since $T_{p}$ is the temperature of the positively charged dust fluid, and $\phi$ is normalized by $k_{\beta} T_{i} / e, x$ is the space variable normalized by $\lambda_{D 1}=\left(k_{B} T_{i} / 4 \pi N_{0} Z_{n} e^{2}\right)^{\frac{1}{2}}, t$ is the time variable normalized by $\omega_{p^{2}}^{-1}=\left(m_{2} / 4 \pi N_{0} Z_{n}^{2} e^{2}\right)^{\frac{1}{2}}$, where $\sigma_{d}=\left(T_{p} / T_{i} Z_{p}\right)$, $\sigma_{e}=\left(T_{e} / T_{i}\right), \quad \mu=\left(\frac{n_{0} Z_{p}}{N_{0} Z_{n}}\right), \mu_{e}=\left(\frac{n_{e 0}}{N_{0} Z_{n}}\right)$, and $\mu_{3 i}=\left(\frac{n_{i 0}}{N_{0} Z_{n}}\right) . \Omega_{1}$ and $\Omega_{2}$ are the positive and negative charged dust cyclotron frequencies normalized to plasma frequency.

\section{NONLINEAR ANALYSIS}

According to the general method of reductive perturbation theory (RPT), we introduce the slow(3) stretched co-ordinates:

$\tau=\varepsilon^{\frac{3}{2}} t$

$\varsigma=\varepsilon^{\frac{1}{2}}(l x+m y+\Gamma z-\lambda t)$

where $\varepsilon$ is a small dimensionless expansion parameter and $\lambda$ is the wave speed normalized by $C_{s} . l$, mand $\Gamma$ are the directional cosines of the wave vector $k$ along the $x, y a n d z$ axis. All physical quantities appearing in (2.1) are expanded as power series in $\varepsilon$ about their equilibrium values as:

$$
\begin{aligned}
& n=1+\varepsilon n_{1}+\varepsilon^{2} n_{2}+\varepsilon^{3} n_{3} \ldots, \\
& u_{x}=\varepsilon u_{x 1}+\varepsilon^{2} u_{x 2}+\varepsilon^{3} u_{x 3}+\ldots, \\
& u_{y}=\varepsilon^{\frac{3}{2}} u_{y 1}+\varepsilon^{2} u_{y 2}+\varepsilon^{\frac{5}{2}} u_{y 3}+\ldots, \\
& u_{z}=\varepsilon^{\frac{3}{2}} u_{z 1}+\varepsilon^{2} u_{z 2}+\varepsilon^{\frac{5}{2}} u_{z 3}+\ldots, \\
& N=1+\varepsilon N_{1}+\varepsilon^{2} N_{2}++\varepsilon^{3} N_{3} \ldots, \\
& v_{x}=\varepsilon v_{x 1}+\varepsilon^{2} v_{x 2}+\varepsilon^{3} v_{x 3}+\ldots, \\
& v_{y}=\varepsilon^{\frac{3}{2}} v_{y 1}+\varepsilon^{2} v y_{2}+\varepsilon^{\frac{5}{2}} v_{y 3} \ldots, \\
& v_{z}=\varepsilon^{\frac{3}{2}} v_{z 1}+\varepsilon^{2} v_{z 2}+\varepsilon^{\frac{5}{2}} v_{z 3} \ldots, \\
& p=1+\varepsilon p_{1}+\varepsilon^{2} p_{2}+\varepsilon^{3} p_{3}+\ldots, \\
& \phi=\varepsilon \phi_{1}+\varepsilon^{2} \phi_{2}+\varepsilon^{3} \phi_{3}+\ldots .
\end{aligned}
$$

The charge-neutrality condition in the dusty plasma is always maintained through the relation

$$
1-\mu+\mu_{e}-\mu_{i}=0 \text {. }
$$

We impose the boundary conditions that as: $|\zeta| \rightarrow \infty, n=N=1, p=1, u=v=0, \phi=0$.

Substituting (3.1) and (3.2) into (2.1)-(2.3) and equating coefficients of like powers of $\varepsilon$. Then, from the lowest-order equations in $\varepsilon$, the following results are obtained:

$$
\begin{aligned}
& n_{1}=\frac{l^{2} \phi_{1}}{\lambda^{2} \rho-l^{2} \gamma \sigma_{d}}, u_{x 1}=\frac{l \lambda \phi_{1}}{\lambda^{2} \rho-l^{2} \gamma \sigma_{d}}, \\
& u_{y_{1}}=-\frac{\Gamma \lambda^{2} \rho}{\left(\lambda^{2} \rho-l^{2} \gamma \sigma_{d}\right) \Omega_{1}} \frac{\partial \phi_{1}}{\partial \zeta}, \\
& u_{z_{1}}=\frac{m \lambda^{2} \rho}{\left(\lambda^{2} \rho-l^{2} \gamma \sigma_{d}\right) \Omega_{1}} \frac{\partial \phi_{1}}{\partial \zeta},
\end{aligned}
$$$$
p_{1}=\frac{l^{2} \gamma \phi_{1}}{\lambda^{2} \rho-l^{2} \gamma \sigma_{d}}
$$ 
$N_{1}=-\frac{l^{2} \phi_{1}}{\lambda^{2}}, v_{x 1}=-\frac{l \phi_{1}}{\lambda}$,

$v_{y_{1}}=-\frac{\Gamma}{\Omega_{2}} \frac{\partial \phi_{1}}{\partial \zeta}, v_{z_{1}}=-\frac{m}{\Omega_{2}} \frac{\partial \phi_{1}}{\partial \zeta}$.

Poisson's equation gives the linear dispersion relation $\frac{\mu_{1} l^{2}}{\lambda^{2} \rho-l^{2} \gamma \sigma_{d}}+\frac{l^{2}}{\lambda^{2}}-\mu_{i}-\mu_{e} \sigma_{e}=0$,

The next-order of the perturbation gives:

$\frac{\partial n_{1}}{\partial \tau}-\lambda \frac{\partial n_{2}}{\partial \zeta}+\ln _{1} \frac{\partial u_{x 1}}{\partial \zeta}+$

$l \frac{\partial u_{x 2}}{\partial \zeta}+m \frac{\partial u_{y 2}}{\partial \zeta}+\Gamma \frac{\partial u_{z 2}}{\partial \zeta}=0$

$-\lambda \rho \frac{\partial u_{x 2}}{\partial \zeta}+\rho \frac{\partial u_{x 1}}{\partial \tau}+l \rho u_{x 1} \frac{\partial u_{x 1}}{\partial \zeta}-l \sigma_{d} n_{1} \frac{\partial P_{1}}{\partial \zeta}+$

$l \sigma_{d} \frac{\partial P_{2}}{\partial \zeta}+l \frac{\partial \phi_{2}}{\partial \zeta}=0$

$-\Omega_{1} u_{z 3}+m \sigma_{d} \frac{\partial P_{2}}{\partial \zeta}-m \sigma_{d} n_{1} \frac{\partial P_{1}}{\partial \zeta}$

$+m \frac{\partial \phi_{2}}{\partial \zeta}-\lambda \rho \frac{\partial u_{y 2}}{\partial \zeta}=0$

$\Omega_{1} u_{y 3}-\Gamma \sigma_{d} n_{1} \frac{\partial P_{1}}{\partial \zeta}+\Gamma \sigma_{d} \frac{\partial P_{2}}{\partial \zeta}$

$-\lambda \rho \frac{\partial u_{z 2}}{\partial \zeta}+\Gamma \frac{\partial \varphi_{2}}{\partial \zeta}=0$,

$\frac{\partial P_{1}}{\partial \tau}-\lambda \frac{\partial P_{2}}{\partial \zeta}+l u_{x 1} \frac{\partial P_{1}}{\partial \zeta}+l \gamma P_{1} \frac{\partial u_{x 1}}{\partial \zeta}$

$+l \gamma\left(\frac{\partial u_{x 2}}{\partial \zeta}+\frac{\partial u_{z 2}}{\partial \zeta}\right)=0$

$u_{y 2}=\frac{m \lambda^{3} \rho^{2}}{\left(\lambda^{2} \rho-\ell^{2} \gamma \sigma_{d}\right) \Omega_{1}^{2}} \frac{\partial^{2} \phi_{1}}{\partial \zeta^{2}}$,

$u_{z 2}=\frac{\Gamma \lambda^{3} \rho^{2}}{\left(\lambda^{2} \rho-L^{2} \gamma \sigma_{d}\right) \Omega_{1}^{2}} \frac{\partial^{2} \phi_{1}}{\partial \zeta^{2}}$,

For Positive dust plasma and

$\frac{\partial N_{1}}{\partial \tau}-\lambda \frac{\partial N_{2}}{\partial \zeta}+l \frac{\partial}{\partial \zeta}\left(v_{x 1} N_{1}\right)+l \frac{\partial v_{x 2}}{\partial \zeta}$

$+m \frac{\partial v_{y 2}}{\partial \zeta}+\Gamma \frac{\partial v_{z 2}}{\partial \zeta}=0$

$$
\begin{aligned}
& \frac{\partial v_{x 1}}{\partial \tau}+l v_{x 1} \frac{\partial v_{x 1}}{\partial \zeta}-\lambda \frac{\partial v_{x 2}}{\partial \zeta}-l \frac{\partial \varphi_{2}}{\partial \zeta}=0 \\
& -\Omega_{2} v_{z 3}-m \frac{\partial \phi_{2}}{\partial \zeta}-\lambda \frac{\partial v_{y 2}}{\partial \zeta}=0 \\
& -\Omega_{2} v_{y 3}-\Gamma \frac{\partial \phi_{2}}{\partial \zeta}-\lambda \frac{\partial v_{z 2}}{\partial \zeta}=0 \\
& v_{y 2}=\frac{m \lambda}{\Omega_{2}^{2}} \frac{\partial^{2} \phi_{1}}{\partial \zeta^{2}}, v_{z 2}=\frac{\Gamma \lambda}{\Omega_{2}^{2}} \frac{\partial \phi_{1}}{\partial \zeta}
\end{aligned}
$$

And for negative dust plasma and Poisson's equation gives:

$$
\begin{aligned}
& \left(l^{2} \frac{\partial^{2}}{\partial \zeta^{2}}+m^{2} \frac{\partial^{2}}{\partial \zeta^{2}}+\Gamma^{2} \frac{\partial^{2}}{\partial \zeta^{2}}\right) \phi_{1}-\frac{1}{2} \mu_{e} \sigma_{e}^{2} \phi_{1}^{2} \\
& +\frac{1}{2} \mu_{i} \phi_{1}^{2}+\mu_{1} n_{2} \\
& -N_{2}-\mu_{i} \phi_{2}-\mu_{e} \sigma_{e} \phi_{2}=0 .
\end{aligned}
$$

Eliminate the second order perturbed quantities $n_{2}, u_{2}, N_{2}, v_{2}$ and $\phi_{2}$ in equations (3.7-3.11), we derive the $\mathrm{KdV}$ equation;

$$
\frac{\partial \phi_{1}}{\partial \tau}+A \phi_{1} \frac{\partial \phi_{1}}{\partial \xi}+\frac{B}{2} \frac{\partial^{3} \phi_{1}}{\partial \xi^{3}}=0,
$$

where

$$
\begin{gathered}
A=\left(2 l^{2} \lambda\left(l^{2} \gamma \sigma_{d}-\lambda^{2} \rho\right)\left(\rho \mu_{1} \lambda^{4}+\left(\lambda^{2} \rho-l^{2} \gamma \sigma_{d}\right)^{2}\right)\right)^{-1} \\
\left(l^{4} \lambda^{4} \mu\left(-(\gamma-1) \gamma \sigma_{d} l^{2}-2 \lambda^{2} \rho\right)-\left(l^{2} \gamma \sigma_{d}-\lambda^{2} \rho\right)^{3}\right. \\
\left.\left.\left(3 l^{4}+\lambda^{4}\left(\mu_{e} \sigma_{e}^{2}-\mu_{i}\right)\right)\right)\right), \\
\quad\{(3.13) \\
B=2\left(2 \Omega_{1}^{2} \Omega_{2}^{2} \ell^{2}\left(\left(\lambda^{2} \rho-\gamma \ell^{2} \sigma_{d}\right)^{2}+\lambda^{4} \mu \rho\right)\right)^{-1} \\
\left(\lambda^{3} \lambda^{2} \mu \rho^{2} \Omega_{2}^{2}\left(\lambda^{2} \rho\left(\Gamma^{2}+m^{2}\right)-m^{2} \ell^{2} \sigma_{d}\right)\right. \\
\left.+\Omega_{1}^{2}\left(\Omega_{2}^{2}\left(\Gamma^{2}+m^{2}+\ell^{2}\right)-m^{2}\right)\left(\lambda^{2} \rho-\gamma \ell^{2} \sigma_{d} l_{1}^{2}\right)\right),
\end{gathered}
$$

Equation (12) adimts the hall-mark soliton solution.

$$
\phi_{1}=\frac{3 \vartheta}{A} \operatorname{sech}^{2}\left[\left(\frac{\vartheta}{2 B}\right)^{1 / 2} \eta\right]
$$

where $\eta$ is the transformed coordinate with respect to a frame moving with velocity $\vartheta$.

\section{HIGHER ORDER CORRECTION}

As it well know, Equation (3.12) contains the lowestorder nonlinearity and dispersion. Its validity is restricted to waves of only small amplitudes. However, as the wave amplitude ncreases, the width and velocity of a soliton deviate from the prediction 


\section{Progress of Nonlinear Dust Acoustic Waves}

of the KdV equation. In order to describe DAWs with an inlarged amplitude, higher-order effects must be taken into account. In particular, we add to the well known $\mathrm{KdV}$ equation additional fifth order dispersion term as a higher-order one,

$$
\frac{\partial \phi_{1}}{\partial \tau}+A \phi_{1} \frac{\partial \phi_{1}}{\partial \xi}+\frac{B}{2} \frac{\partial^{3} \phi_{1}}{\partial \xi^{3}}+\delta \frac{\partial^{5} \phi_{1}}{\partial \xi^{5}}=0,
$$

where $\delta$ is a smallness parameter. In the case of $\delta$ $=0$, equation (4.1) reduces identically to the well known KdV equation (3.12). Due to the secularities included in the last term of equation (4.1), it could not be solved exactly, e.g. only trivial solutions (travelling waves) were found by classical Lie groupmethod; for a correct solution we rather have to rely on a the perturbation method, in which the secularities embedded in the perturbed term are separated such that the equations in each order of $\delta$ are secular. In the following we introduce another transformation $\eta=\xi-\vartheta \tau$ to recast equation (4.1) is introduced into

$-\vartheta \frac{d \phi_{1}}{d \eta}+A \phi_{1} \frac{d \phi_{1}}{d \eta}+\frac{B}{2} \frac{d^{3} \phi_{1}}{d \eta^{3}}+\delta \frac{d^{5} \phi_{1}}{\partial \eta^{5}}=0$.

In order to solve equation (4.2), a simple method constructed by (Watanabe and Jiang 1993) is introduced for finding a higher-order solitary wave solutions. Accordingly, expanding $\phi_{1}$ and $\vartheta$ as well with respect to the smallness parameter $\delta$,

$$
\begin{gathered}
\phi_{1}=\psi_{0}+\delta \psi_{1}+\delta^{2} \psi_{2}+\delta^{3} \psi_{3}+\ldots \ldots . ., \\
\}(4.3) \\
\vartheta=\vartheta_{0}+\delta \vartheta_{1}+\delta^{2} \vartheta_{2}+\delta^{3} \vartheta_{3}+\ldots \ldots .,
\end{gathered}
$$

Substituting equation (4.3) into equation (4.2) and comparing the coefficients of like power in $\delta$, one arrives at the following numbers of coupled ordinary differential equations:

$$
\begin{aligned}
& \delta^{0}:-\vartheta_{0} \frac{d \psi_{0}}{d \eta}+A \psi_{0} \frac{d \psi_{0}}{d \eta}+\frac{B}{2} \frac{d^{3} \psi_{0}}{d \eta^{3}}=0 \\
& \delta^{1}: L \psi_{1}=\vartheta_{1} \frac{d \psi_{0}}{d \eta}-\frac{d^{5} \psi_{0}}{d \eta^{5}}, \\
& \delta^{2}: L \psi_{2}=\vartheta_{1} \frac{d \psi_{1}}{d \eta}+\vartheta_{2} \frac{d \psi_{0}}{d \eta}-A \psi_{1} \frac{d \psi_{1}}{d \eta}-\frac{d^{5} \psi_{1}}{d \eta^{5}} \\
& \delta^{3}: L \psi_{3}=\vartheta_{3} \frac{d \psi_{0}}{d \eta}+\vartheta_{2} \frac{d \psi_{1}}{d \eta}+\vartheta_{1} \frac{d \psi_{2}}{d \eta}-A \frac{d\left(\psi_{1} \psi_{2}\right)}{d \eta}-\frac{d^{5} \psi_{2}}{d \eta^{5}}
\end{aligned}
$$

where the operator $\mathrm{L}$ represents

$$
L=-\vartheta_{0} \frac{d}{d \eta}+A \psi_{0} \frac{d}{d \eta}+\frac{B}{2} \frac{d^{3}}{d \eta^{3}} \text {. }
$$

In fact, this differential operator is the singlevariable version of the linearized $\mathrm{KdV}$ operator. To obtain the solutions, solving equatios (4.4a-4.4d) successively and subject them to the boundary conditions $\psi_{i}=0(i=0,1,2, \ldots), \frac{d \psi_{i}}{d \eta}=0$, and $\frac{d^{2} \psi_{i}}{d \eta^{2}}=0$ for $\eta \rightarrow \pm \infty$.Equation (4.4a) is fulfilled by a solitary wave solution of the form $\psi_{0}=\psi_{m} \operatorname{sech}^{2}(D \eta)$,

where the soliton amplitude $\psi_{m}$ and the soliton width $D^{-1}$ are

$$
\psi_{m}=\frac{3 \vartheta_{0}}{A} \text { and } D^{-1}=\sqrt{\frac{2 B}{\vartheta_{0}}} \text {. }
$$

Note that equation (4.6) is just a single-soliton solution of the $\mathrm{KdV}$ equation.

In the next order of $\delta$ substituting equation (4.6) into Equation (4.4b), we obtain :

$$
\begin{aligned}
& L \psi_{1}=\operatorname{sech}^{4}(D \eta)\left(\frac{-1440 D^{5} \vartheta_{0}}{A}-\frac{144 B D^{3} \vartheta_{0}^{2}}{A^{2}}\right) \tanh (D \eta)+ \\
& \operatorname{sech}^{6}(D \eta)\left(\frac{2160 D^{5} \vartheta_{0}}{A}+\frac{216 B D^{3} \vartheta_{0}^{2}}{A^{2}}+\frac{54 D \vartheta_{0}^{3}}{A^{2}}\right) \tanh (D \eta) \\
& \left.+\operatorname{sech}^{2}(D \eta)\left(\frac{96 D^{5} \vartheta_{0}}{A}-\frac{6 D \vartheta_{0} \vartheta_{1}}{A}\right) \tanh (D \eta) .(4.7) 15\right)
\end{aligned}
$$

Equation (4.2) is a third-order linear differential equation associated with inhomogeneous terms on the right-hand side. The homogeneous equation, $L \psi_{1}=0$, satisfying the boundary conditions, has a solution that is proportional to $\operatorname{sech}^{2}(D \eta) \tanh (D \eta)$. Let us assume here a solution of the above equation of the form: $\psi_{1}=\mu_{1} \operatorname{sech}^{2}(D \eta)+\mu_{2} \operatorname{sech}^{4}(D \eta)$

with $\mu_{1}$ and $\mu_{2}$ representing constants, which can readily be proved by re-substitution. Then, it is easily to observe that the coefficient of $\operatorname{sech}^{2}(D \eta)$ on the left-hand side cancels out and $L \psi_{1}$ is expressed in terms of $\operatorname{sech}^{4}(D \eta)$ and $\operatorname{sech}^{6}(D \eta)$. In that case, it will be obviously that the coefficient of $\operatorname{sech}^{2}(D \eta)$ on the right-hand side should vanish, 
which leads to the first order correction of the velocity

$$
\vartheta_{1}=\frac{4 \vartheta_{0}^{2}}{B^{2}}
$$

The coefficients in equation (4.8) can be found to constitute the solution:

$$
\psi_{1}=\frac{-30 \vartheta_{0}^{2}}{A B^{2}} \operatorname{sech}^{2}(D \eta)+\frac{45 \vartheta_{0}^{2}}{\mathrm{AB}^{2}} \operatorname{sech}^{4}(D \eta) \text {. }
$$

In order to evaluate next orders of $\delta$, we introduce equations (4.6),(4.7) and (4.10) into (4.4c$4.4 \mathrm{~d})$, and after algebric manipulation we can get,

$$
\begin{aligned}
& \vartheta_{2}=0 \\
& \psi_{2}=\frac{90 \vartheta_{0}^{3}}{A B^{4}} \operatorname{sech}^{2}(D \eta)-\frac{1395 \vartheta_{0}^{3}}{A B^{4}} \operatorname{sech}^{4}(D \eta) \\
& +\frac{1395 \vartheta_{0}^{3}}{A B^{4}} \operatorname{sech}^{6}(D \eta) . \\
& \vartheta_{3}=0, \\
& \psi_{3}=\frac{-3708 \vartheta_{0}^{4}}{A B^{6}} \operatorname{sech}^{2}(D \eta)+\frac{31554 \vartheta_{0}^{4}}{A B^{6}} \operatorname{sech}^{4}(D \eta) \\
& -\frac{99324 \vartheta_{0}^{4}}{A B^{6}} \operatorname{sech}^{6}(D \eta) \\
& +\frac{74493 \vartheta_{0}^{4}}{A B^{6}} \operatorname{sech}^{8}(D \eta) .
\end{aligned}
$$

Finally, combining now equations. (4.6), (4.9), (4.10), (4.11) and (4.12), we can obtain the solution of the perturbed $\mathrm{KdV}$ equation. This solution are expressed by power series of lower-order solutions thus eliminating secularities, that the wave velocity depends on $\delta$ only to the first order, while the shape (profile of potential) of solitary wave depends on all orders of $\delta$.

\section{DISCUSSIONS and CONCLUSIONS:}

To make the results physically relevant, numerical calculations were performed referring to typical dusty plasma parameters as given in Ref. [29,30]. The effect of $\rho$ and $v$ on the the higherorder potential amplitude $\psi_{m}$ in Figs.1. It is seen that the present system supports comprisive solitons. However, the effect of $\Omega_{2}$ and $\sigma_{d}$ on the higher order solitons amplitude are shown in Fig.2. it is noticed that, $\rho$ and $v$ increases the soliton amplitude but $\Omega_{2}$ and $\sigma_{d}$ is found to decrease the soliton amplitude. On the other hand, one of our motivations was to study the effect of smallness perturbation parameter $\varepsilon$ on formation of the broadband electrostatic noise. For example, Fig. 3 and Fig. 4 Show that, the perturbation parameter $\varepsilon$ increases the amplitude and decreases the width of higher order soliton and the related electric field. In summary, it has been found that the presence of $\rho$ and $\sigma_{d}$ and $\varepsilon$ would modify the properties of the DAWs and the results presented here should be helpful for understanding salient features of localized electrostatic perturbations in space and laboratory plasma.

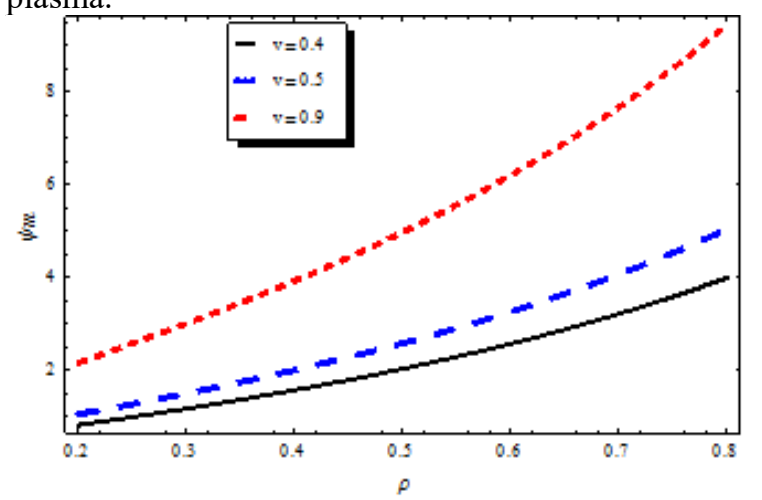

Fig. 1. Variation of the higher-order potential amplitude vs. $\rho$ for $\sigma=0.1$ and $\varepsilon=0.04$. for different values of $v$.

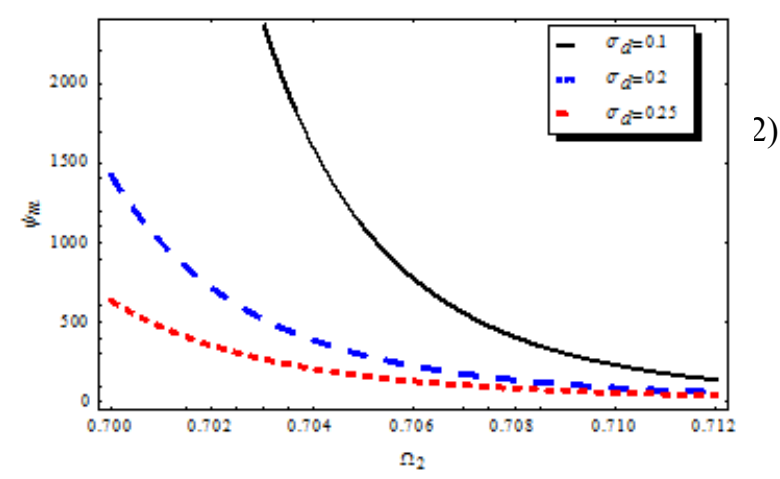

Fig.2 Variation of the higher-order potential amplitude vs. $\Omega_{2}$ for $v=0.04$ and $\varepsilon=0.05$. for different values of $\sigma_{d}$.

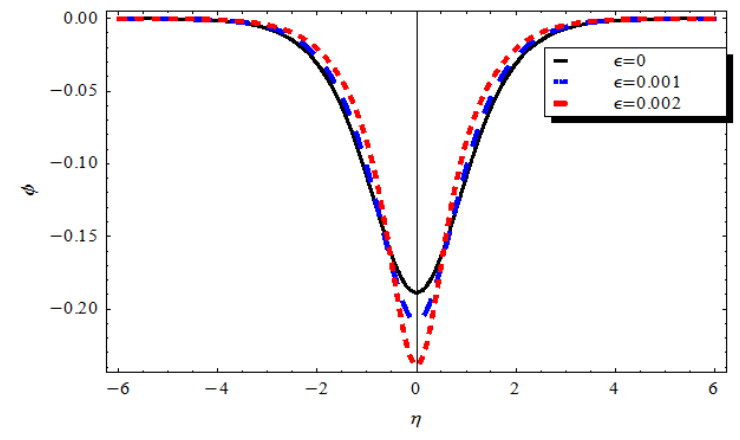


Fig.3 Variation of the higher-order potential $\phi$ vs. $\eta$ for $v=0.08, \delta=0.6$ and $p=0.1$ for different values of $\mathcal{E}$.

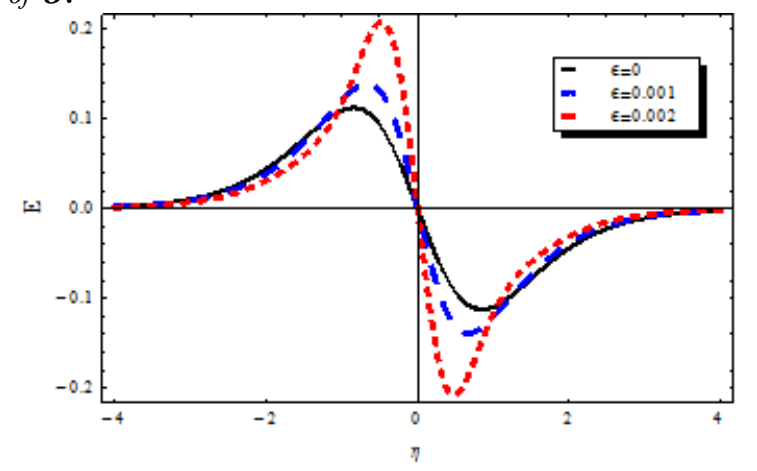

Fig.4 Variation of the associated higher-order electric field structures $E$ vs. $\eta$ for $v=0.08, \delta=0.6$ for different values of $\mathcal{E}$.

\section{Reference}

[1] M. Horanyi, Annu.Rev. Astron. Astrophys. Vol.34, p.383, 1996.

[2] P.K.Shukla, Phys.Plasmas, vol. 8, p.1791, 2001.

[3] A. Barkan, D’Angelo,N. and Merlino, R. L., ibid. vol.73, p. 3093, 1994.

[4] N.N. Rao, P.K. Shukla and M.Y. Yu, Planet. Space Sci. vol. 38, p. 543, 1990.

[5] P. K. Shukla and V. P. Silin , Phys. Scr. Vol. 45 , p. $508,1992$.

[6] A. Barkan , N. DAngelo, and R. L. Marlino, Planet. Space Sci. vol. 44, p. 239, 1996.

[7] F. Melandsø , Phys. Plasmas. Vol. 3, p. 3890, 1996.

[8] A. Homann , A. Melzer , S. Peters and Piel A., Phys. Rev.E vol. 56, p. 7138, 1997.

[9] C. K. Goertz, Rev. Geophys., vol. 27, p.271, 1989.

[10] E. C.Whipple, Rep. Prog. Phys. Vol. 44, p. 1197 1981.

[11]Meyer-Vernet,N.,Astron.Astrophys vol..105, p.98, 1982.

[12] A. Mamun and P. K. Shukla, Geophys. Res. Lett. Vol. 29, p. 1870, 2002.

[13] S A. Elwakil, M T. Attia, M. A. Zahran, E. K. El-Shewy, H G. Abdelwahed, Z. Naturforsch.;
61, no a,p. 316, 2006.

[14] S. A. El-Wakil, M. A. Zahran, E. K. El-Shewy, and A. E. Mowafy, Phys. Scr. Vol.74, p.503 2006.

[15] A. E. Mowafy , E. K. El-Shewy, W. M. Moslem , M. A. Zahran, Phys. Plasma, vol. 15, no. 7,p. $073708,2008$.

[16] F. Sayed and A. A. Mamun, Phys. Plasmas vol.14, p.014501, 2007.

[17] E.K .El-Shewy, Zahran, M.A., Schoepf, K., Elwakil, S.A. PhysicaScripta vol. 78 , no. 2, p. 025501, 2008.

[18] S.K. Zaghbeer, H.H. Salah, N.H. Sheta, E.K. ElShewy, and A. Elgarayhi, J. Plasma Physics, vol. 80, no. 3,P.517-528, 2014.

[19] S.K. Zaghbeer, H.H. Salah, N.H. Sheta, E.K. ElShewy, and A. Elgarayhi, Astrophys Space Sci, vol. 353,P.493-500, 2014.

[20] M T. Attia, M. A. Zahran, E. K. El-Shewy, A. E. Mowafy, Z. Natur- forsch. Vol. 65a, 91, 2010.

[21]H. Washini and T. Taniuti, Phys. Rev. Lett. Vol.17, p. 996, 1966.

[22]S.K. El-Labany J. Plasma Phys. 54, 295 (1995).

[23]S.A. Elwakil, E.K. El-Shewy, and M.A. Zahran, Chaos, Solitons, and Fractals vol.22, p. 13, 2004.

[24]E.K. El-Shewy, Chaos, Solitons, and Fractals vol. 26, p. 1073, 2005.

[25]E.K. El-Shewy, Chaos, Solitons, and Fractals vol. 31, 1020, 2007a.

[26] S.K. Elwakil, E.K. El-Shewy, and R. Sabry, Int. J. Nonlinear Sci. Numer. Simul. Vol. 5, p. 403, 2004.

[27] M.Horanyi, G. E. Mor... 11, and E. Grun, Mechanism for the accelera- tion and ejection of dust grains from Jupiter's magnetosphere,

Nature,vol. 363, p. 144, 1993.

[28] V. E. Fortov, A. P. Nefedov, O. S. Vaulina, A. M. Lipaev, V. I. Molotkov., et al., J. Exp. Theor. Phys.,vol. 87, p.1087-1097, 1998.

[29] O.Havens, et al., Geophys. Res. Lett. Vol. 28, p. 1419, 2001.

[30] B.Smiley, et al., J. Geophys Res. Vol. 108, doi:10.1029/2002JD002425, 2003. 